\title{
Prison Identification Technology Research Based on Iris Image Recognition
}

\author{
${ }^{1}$ Lv Hanfei and ${ }^{2}$ Jiang Congfeng \\ ${ }^{1}$ Department of Information Technology and Management \\ Zhejiang Police Vocational Academy \\ Hangzhou, Zhejiang Province, China \\ ${ }^{2}$ School of Computer Science and Technology \\ Hangzhou Dianzi University \\ Hangzhou, Zhejiang Province, China \\ lhf_b@sina.com,cjiang@hdu.edu.cn
}

\begin{abstract}
In this paper we study one prison identification method based on iris image recognition to meet the real-time identification requirements for prison management. The identification method for online real-time acquisition personnel iris image using preprocessing algorithm to improve the quality of image for subsequent feature extraction. Moreover, we use the coarse-to-fine approach to precisely extract the iris information. We combine the edge extraction method and the connected domain characteristics of multistage de-noising method to locate the inner and outer boundary. Finally we use image multiple features template matching method to recognize the iris information. The experimental results show that the proposed algorithm can correctly identify the iris image quickly. It can meet the requirements of the prison site management.
\end{abstract}

Keywords: Iris Recognition, Pre-precossing, Prison Management System

\section{Introduction}

With the rapid development of electronic information and modern management, the demand for informazation and security management is growing urgently. According to the prison informazation this paper begins to study the requirements of the prison management system. At present there are several identification information management technologies. The popular methods include face recognition, fingerprint recognition and iris recognition. Iris recognition is one of the most promising bio-metric recognition method. It has several good features: high recognition rate, non-contact and fraudulence proof. It has important application prospects because of high security and high accuracy $[1,2]$. But it is not widely applied in the prison for some reasons. The prison environment is complex and the personnel of image acquisition is complicated. The external environment affects the correct identification of the iris, such as field uneven light, background light intensity and angle variety. The location of the personnel iris image acquisition is not fixed. The blink will cause the image blurring and the eyelid or eyelash interference is an obstacle. In order to meet the real-time requirements of field application of prison management system, the iris correct recognition rate, recognition speed and adaptability have some limitations. 


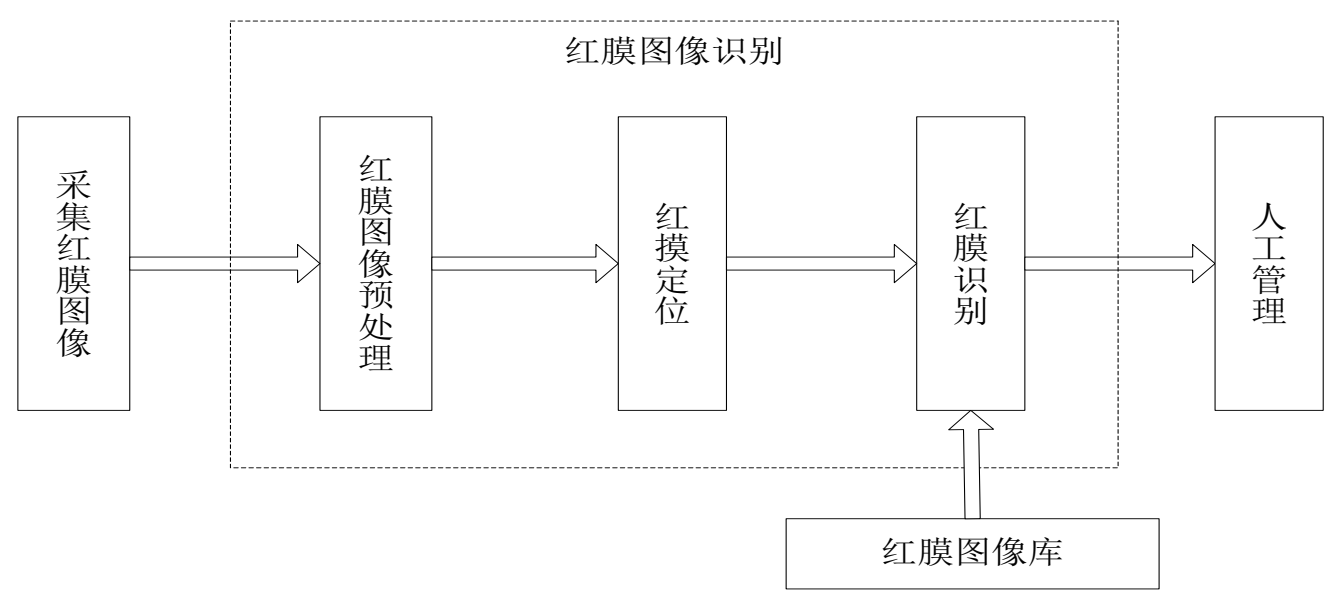

Figure 1. Iris Recognition System

For the recognition and application of iris image, domestic and foreign scholars have studied related theories and methods $[1,2]$. They have been used for the important place bank, airport, judicial departments and other security. But because of the influence of actual complex factors, iris recognition technology promotion and application in the field of prison informatization and modernization is restricted. We need the effective technology to meet the safety management of the prison system. Aiming at the urgent demand we should put forward the research of prison technology of identity recognition based on iris image recognition.

\section{Iris Pre-Processing}

At pre-processing step, our task is to remove noise and unwanted area in the iris image. The iris image quality is not so good for the influence of image acquisition environment. In order to reduce the image noise and improve quality of iris image, we must use the noise reduction algorithm in pre-processing step.

From Figure 2(c), the edge extraction experiment results show that the noise reduction approach can control noise effectively and keep the iris boundary data better [13]. And it gets excellent detection effect of boundary for the subsequent iris precise localization at the same time.

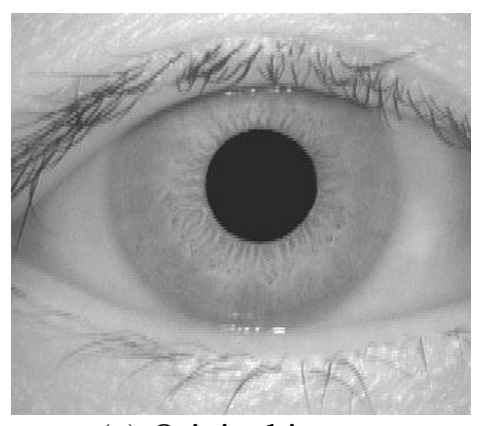

(a) Original image

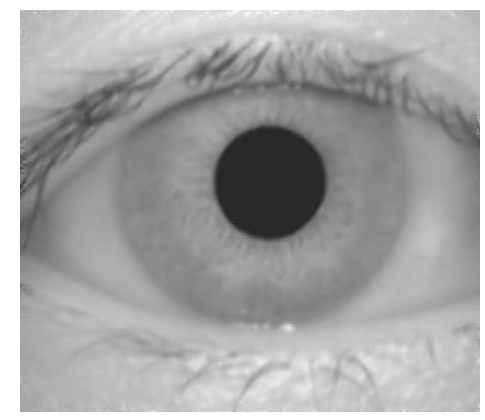

(b) Pre-processing image

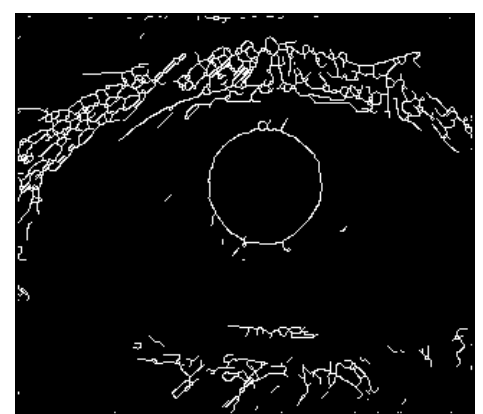

(c) Canny edge detection of pre-processing

Figure 2. Iris Image after Pre-processing

\section{Iris Localization}

To determine the iris outer boundary and inner boundary, we must distinguish the boundary of the iris and sclera, the boundary of the iris and pupil in eye images. 


\subsection{Iris Inner Boundary Localization}

The inner boundary of the iris includes the limbus part at the junction of iris and pupil. The gray of iris inner boundary vary apparently. When the person does not blink the eyelash and eyelid occlusion trouble exists. The contrast of iris image is higher than other parts of the eye in the infrared illumination. The localization for the inner boundary of the iris is easy. Firstly we adopt the traditional edge detection method for iris inner boundary detection of image after pre-preocessing. Secondly we use the connected domain characteristics of multistage denoising approach to remove the eyelash, eyelid and other interfering parts of the eye. Thirdly we adopt the circle fitting approach based on the precise location of the inner iris boundary to locate the circle center. Finally we do the localization work of the iris inner boundary.

We use the Canny operator for iris boundary extraction. The Canny operator is used to extract the iris inner edge and it can preserve the iris inner edge well. And the detail characteristics are suitable for the follow-up precise localization.

\subsubsection{Connected Domain Feature Denoising}

We extract the iris inner boundary data based on boundary detection of iris image. It is good to ensure the accuracy of the inner boundary location of the iris. We remove the background region inconsistent with the inner boundary character first. Then we highlight the iris inner boundary region. And we gradually reduce the interference of the background and highlight the iris inner boundary data with feature set filtering approach. We adopt area feature filtering method, distance feature filtering method, deburring algorithm to do the connected domain feature denoising.

\subsubsection{Iris Inner Boundary Localization}

After feature set filtering, the iris inner edge has been accurately extracted. We use the method of circle fitting to get the center of inner boundary. Then we locate the iris inner boundary in the original iris image. Then we locate the inner boundary of iris image and we show the localization result in Figure 3.

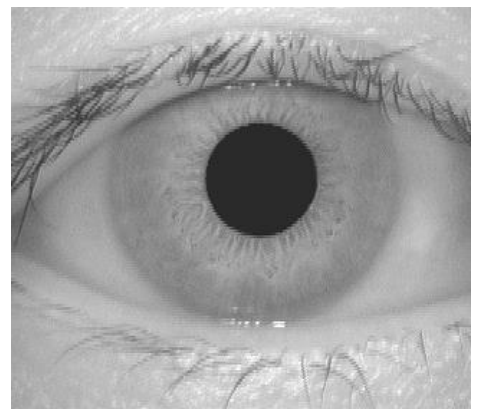

(a) iris image

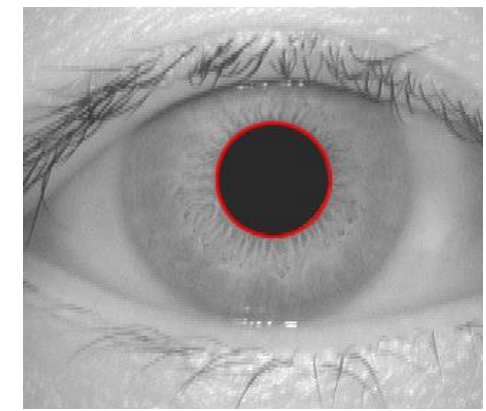

(b) iris inner boundary localization

Figure 3. Iris Inner Boundary Localization 


\subsection{Iris Outer Boundary Localization}

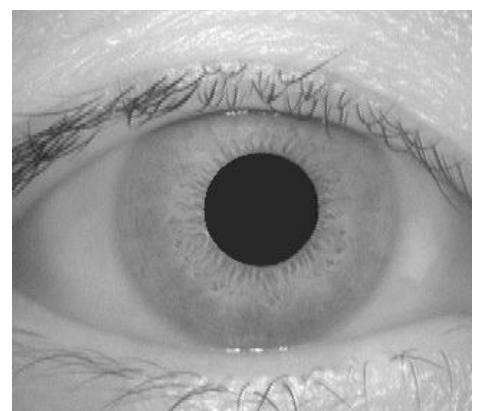

(a) Iris image

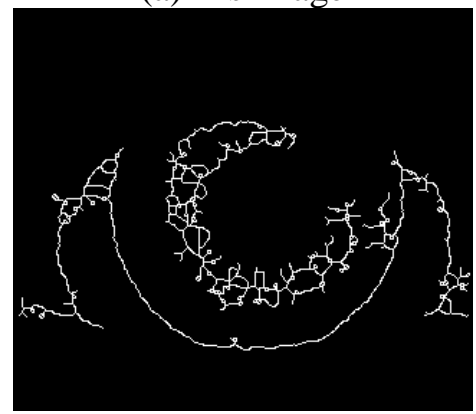

(c) Region feature filtering

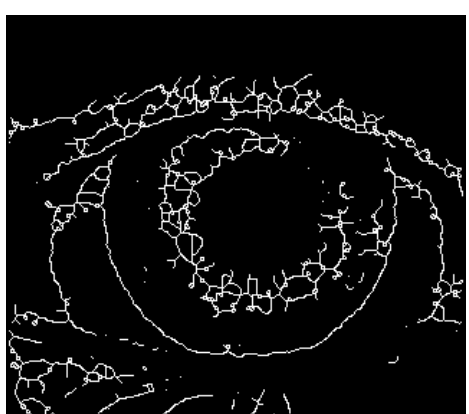

(b) LOG boundary detection

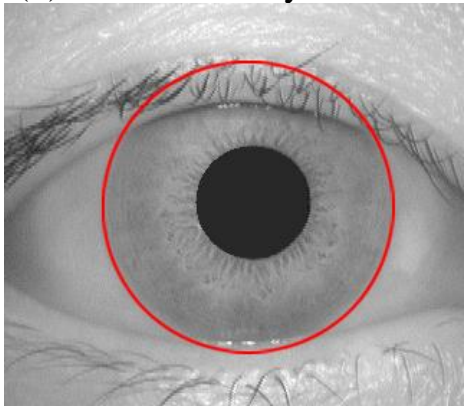

(d) Scleral boundary localization

Figure 4. Iris Outer Boundary Localization

The outer boundary of iris includes the sclera limbus edge and a part of the iris. The edge is unclear and the part of the transition band is wide. At the same time it is hard to get the accurate outer iris boundary with the interference of eyelids and eyelashes. It is difficult to extract the iris outer boundary features because they are fuzzy. But it is easy to distinguish the iris and sclera for the reason of the obvious contrast. And we use one segmentation method which combines OTSU and the contrast to discern between the iris and sclera in the first step. We adopt the LOG operator to extract the weak iris outer boundary data in the next step. Then the features set filtering approach is used to cut off the interference outside the region of iris outer boundary. The last step is to achieve the localization of the iris outer boundary.

In the iris image after features set filtering, the main interference region includes sclera, canthus, eyelid and other regions in the iris image. In iris image the features of outer boundary are different from the inner boundary. It is not a closed region for outer boundary. The sclera is just a small connected region. The sclera interference can be removed after we calculate the closed region number. We find that most of the target domains for the eyelid and canthus connected with the outer boundary are independent neighborhood. We can use the similar approach in 3.1.2 to fit the outer boundary from the target point of the independent neighborhood. The result is shown in Fig. 7. Only the fitting target of solitary neighborhood without neighborhood is selected for subsequent processing.

\section{Iris Template Matching Method}

Matching is the process of calculating the degree of similarity between the input test image and training image from database. At present, there are several excellent iris recognition matching algorithm as below:

(1) The classic method based on texture analysis;

(2) The method based on phase analysis; 
(3) The method based on zero-crossing detection;

(4) The method based on image matching.

In this paper, we use the method based on image matching. We calculate the similarity between the iris image acquisition part and the template in the template library. The template image of maximum similarity is the recognition result. If the maximum similarity is very small, it means that the acquisition of iris image is for the new employees. We need to do the manual confirmation of iris image acquisition for new employees. The new iris image can be added to the template library, Similarly we can also delete the unused personnel iris image. For the final similarity character, the correlation function for identifying image and the template image, the calculation formula is as below:

$$
R_{i}=\frac{\sum_{x=1}^{M} \sum_{y=1}^{N} F(x, y) \times T_{i}(x, y)}{\sqrt{\sum_{x=1}^{M} \sum_{y=1}^{N}[F(x, y)]^{2}} \sqrt{\sum_{x=1}^{M} \sum_{y=1}^{N}\left[T_{i}(x, y)\right]^{2}}}
$$

$i \in[1, J], \quad x \in[1, M], \quad y \in[1, N]$

Among them, $F(x, y)$ represents the collected iris image to be recognized. $T_{i}(x, y)$ represents the template image. $\mathrm{J}$ is iris template number. $\mathrm{M}$ is for iris image row and $\mathrm{N}$ is for iris image column. $\mathrm{R}_{\mathrm{i}}$ represents the similarity acquisition image and template image. $0 \leq R_{i} \leq 1$. When the recognition of iris image and template image are matching, the similarity value is greater. At the same time, because the template matching algorithm of iris recognition time and template library is directly related to the size, in order to improve the recognition time and accelerate the response, this paper proposes classification of template library, according to the different kinds of designs different template library, and all kinds of templates are representative. The number of them should not be too much to improve the iris recognition speed from the strategy.

\section{Experiments}

\subsection{Experiment Environment Introduction}

We used CASIA-V1.0, CASIA-V2.0-Device1 and CASIA-V3.0-Twins databases to evaluate the performance of our iris recognition system developed for prison application. (http://www.cbsr.ia.ac.cn/IrisDatabase.htm). The tools include openCV and Visual $\mathrm{C}++6.0$ for the development. The computer configuration is as below: intel i5 3230@2.6GHz CPU and 4G RAM. 


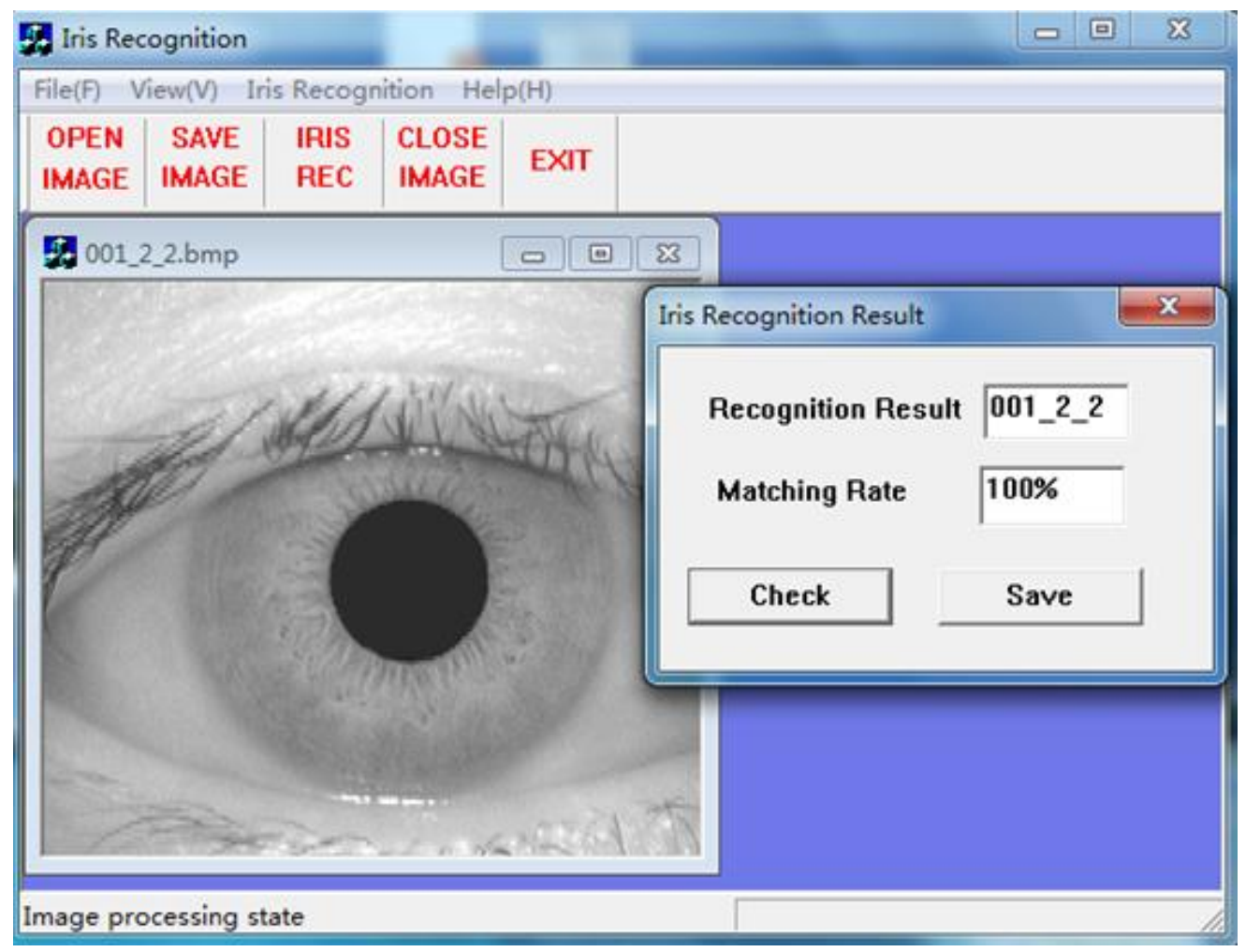

\subsection{Recognition Ratio Contrast}

We use PIM (Prison Identification Method) to represent the method for iris recognition in this paper. We mainly use the CRR (Correct Recognition Rate) and EER(Equal Error Rate) to evaluate the performance of several different methods in contrast. The experimental results are listed in table I. The performance of PIM is better than other two approaches obviously. At the same time, PIM is a fast method. We select 180 different iris images to do the experiment from the three databases (60 iris images from each database). And the average time cost for the total recognition is only $1.21 \mathrm{~s}$. It can meet the requirements of the prison application.

Table 1. Experimental Results of EER and CRR

\begin{tabular}{|c|c|c|c|c|}
\hline & Iris Lib & Sample Num & CRR & EER \\
\hline Daugman & \multirow[t]{3}{*}{ CASIA-1.0 } & \multirow[t]{3}{*}{756} & $97.38 \%$ & $2.62 \%$ \\
\hline Wildes & & & $97.16 \%$ & $2.84 \%$ \\
\hline PIM & & & $98.64 \%$ & $1.36 \%$ \\
\hline Daugman & \multirow[t]{3}{*}{ CASIA-2.0-Device1 } & \multirow[t]{3}{*}{1200} & $96.49 \%$ & $3.51 \%$ \\
\hline Wildes & & & $96.36 \%$ & $3.64 \%$ \\
\hline PIM & & & $97.27 \%$ & $2.73 \%$ \\
\hline Daugman & \multirow[t]{3}{*}{ CASIA-3.0-Twins } & \multirow[t]{3}{*}{3183} & $94.64 \%$ & $5.36 \%$ \\
\hline Wildes & & & $95.65 \%$ & $4.35 \%$ \\
\hline PIM & & & $96.32 \%$ & $3.66 \%$ \\
\hline
\end{tabular}

\section{Conclusion}

This paper presents the study of prison technology of identity recognition based on iris image recognition. The iris recognition technology is suitable for the application of prison management system. We firstly use the image pre-processing algorithm to improve image quality. Secondly, we use the coarse-to-fine location method to make up the image defect and remove the interference. And we can accurately extract the iris information. Finally we use the template matching method 
to meet the fast, real-time requirements of the prison management application. Experimental results show that the proposed algorithm can correctly identify the iris image quickly. It also has certain stability and adaptability to meet the demand of the prison site management.

\section{Acknowledgements}

We greatly appreciate the funding supports of this work by the Commonweal Project Funds of Zhejiang Science \& Technology Department (Grant No. 2013C31115), the Zhejiang Provincial Natural Science Foundation(Grant No. Y13F020205, LY13F020047) and the Open Project of Zhejiang Provincial Engineering Center(Grant No. 2012E1002315).

\section{References}

[1] A. G. Gale1 and S. S. Salankar, "A Review On Advance Methods Of Feature Extraction In Iris Recognition System", IOSR Journal of Electrical and Electronics Engineering (IOSR-JEEE) eISSN: 2278-1676, p-ISSN: 2320-3331, (2014), pp. 65-70.

[2] A. S. Alotaibi and M. A. Hebaishy, "Increasing the Efficiency of Iris Recognition Systems by Using Multi-Channel Frequencies of Gabor Filter", Journal of Remote Sensing Technology, vol. 2, no. 1, February (2014), pp. 98-107.

[3] J G. Daugman, "High Confidence Visual Recognition of Persons by a Test of Statistical Independence", IEEE Trans on Pattern Analysis and Machine Intelligence, vol. 15, no. 11, (1993), pp. 1148-1161.

[4] R P. Wildes, "Iris Recognition: An Emerging Biometric Technology", Proc of the IEEE, vol. 85, no. 9, (1997), pp. 1348-1363.

[5] W W. Boles and B. Boashash, "A Human Identification Technique Using Images of the Iris and Wavelet Transform”, IEEE Trans on Signal Processing, vol. 46, no. 4, (1998), pp. 1185-1188.

[6] Y. Wang, Y. Zhu and T. Tan, "Biometrics Personal Identification Based on Iris Pattern", Acta Automatica Sinica, vol. 28, no. 1, (2002), pp. 1-10.

[7] L. Ma, T. Tan, Y. Wang and D. Zhang, "Efficient Iris Recognition by Characterizing Key Local Variations", IEEE Trans on Signal Processing, vol. 46, no. 4, (1998), pp. 1185-1188.

[8] J. Sun, Z. Li, J. Yang, B. Xu and X. Zhou, "Pretreatment method in auto car tire number recognition", Journal of Jiangsu University (Natural Science Edition), vol. 26, no. 5, (2005), pp. 401-404.

[9] Y. Guo, J. Gu, C. Gao and M. Xu, "Encoding Algorithm for Iris Image Based on Gabor Filtering", OptoElectronic Engineering, vol. 33, no. 4, (2006), pp. 35-38.

[10] W. Yuan, W. Liu and L. Ke, "The Parameter Selection of Gabor Filter for Iris Feature Extraction", Journal of Optoelectroincs. Laser, vol. 19, no. 9, (2008), pp. 1235-1239.

[11] J. Zhang, E. Liu, J. Wan, Y. Ren, M. Yue and J. Wang, "Implementing Sparse Matrix-Vector Multiplication with QCSR on GPU”, Applied Mathematics \& Information Sciences, vol. 7, no. 2, (2013), pp. 473-482.

[12] H. Lv and C. Jiang, "One Hamming Distance Deviation Matching Approach for Iris Recognition", International Journal of IJSIA. IEEE International Journal of Security and its Applications, (2014), vol. 8.

[13] J. Canny, “A Computational Approach to Edge Detection”, IEEE Transaction on Pattern Analysis and Machine Intelligence, vol. 8, no. 6, (1986), pp. 679-698.

[14] J. Daugman, "New Methods in Iris Recognition", IEEE Transactions on Systems, Man and Cybernetics, vol. 37, no. 5, (2007), pp. 1167-1175.

[15] Y. Liu, X. Li, N. Wang, Q. Wang and W. Peng, "An improved iris location algorithm based on sampling to special regions of interesting", Acta Photonica Sinica, vol. 37, no. 6, (2008), pp. 1277- 1280.

[16] P. A. Kumari and G. J. Suma, "A Novel Multimodal Bio metric Scheme for Personal Authentication", International Journal of Research in Engineering \& Technology, vol. 2, no. 2, (2014), pp. 55-66. 


\section{Authors}

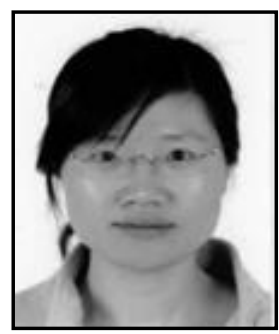

Lv Hanfei, She is an associate professor of Department of Information Technology and Management, Zhejiang Police Vocational Academy. Before joining Zhejiang Police Vocational Academy, she was a master student of Zhejiang University from 2002 to 2005 . Her current research areas include image processing, power aware computing system and its applications in computing systems.

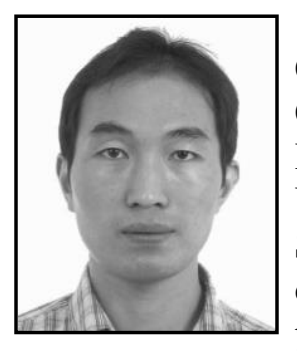

Congfeng Jiang, $\mathrm{He}$ is an associate professor of School of Computer Science and Technology, Hangzhou Dianzi University, China. He is with the Grid and Services Computing Lab in Hangzhou Dianzi University. Before joining Hangzhou Dianzi University, he was a $\mathrm{PhD}$ candidate in Huazhong University of Science and Technology from 2002 to 2007 . He received his $\mathrm{PhD}$ degree in 2007. His current research areas include power aware computing system, virtualization, grid computing, etc. $\mathrm{He}$ is a member of ACM, IEEE, and China Computer Federation (CCF). 